Article

\title{
Synthesis and Evaluation of Poly(hexamethylene-urethane) and PEG-Poly(hexamethylene-urethane) and Their Cholesteryl Oleyl Carbonate Composites for Human Blood Biocompatibility
}

\author{
Mei Fen Shih ${ }^{1, \dagger}$, Min Da Shau ${ }^{2, \dagger}$, Cheng Chih Hsieh ${ }^{3}$ and Jong Yuh Cherng ${ }^{4, *}$
}

1 Department of Pharmacy, Chia Nan University of Pharmacy and Science, 60 Erh-Jen Rd.,

\author{
Sec. 1,Tainan 717, Taiwan
}

2

Department of Life Science, Chia Nan University of Pharmacy and Science, 60 Erh-Jen Rd., Sec. 1, Tainan 717, Taiwan

3

Department of Pharmacy Practice, Tri-service General Hospital, Taipei 114, Taiwan

4

Department of Chemistry and Biochemistry, National Chung Cheng University, 168 University Rd., Chia-Yi 621, Taiwan

$\uparrow$ These authors contributed equally to this work.

* Author to whom correspondence should be addressed; E-Mail: jycherng@yahoo.com;

Tel.: +886-5-272-0411, ext.: 66416; Fax: +886-05-272-1040.

Received: 19 August 2011; in revised form: 19 September 2011 / Accepted: 22 September 2011 / Published: 28 September 2011

Abstract: Two new urethane-based acrylates (UAA and PEG-UAA) were synthesized as polymer blocks. The chemical composition of the two monomers was confirmed by IR and NMR. After cross-linking these blockers by radical polymerization, "hexamethylene PU" [poly(hexamethylene-urethane)] and "PEG-hexamethylene PU" [PEG-poly(hexamethylene-urethane)] were obtained. The platelet adhesion and platelet activation of these polymers were evaluated in the presence of Platelet Rich Plasma (PRP) blood. The relative blood clotting indexes of the polymers were determined to measure their capability of reducing thrombogenicity. The hemolysis of red blood cells was also assessed to examine the haemocompatibility of the polymers. The hexamethylene PU and PEG-hexamethylene PU showed less platelet adhesion, platelet activation, blood clotting and hemolysis than a commercial PU (Tecoflex). The liquid crystal molecule, cholesteryl oleyl carbonate (COC), showed further improved biocompatibility to human blood, after COC was embedded in the PU polymers. PEG-hexamethylene PU $+10 \%$ COC demonstrated the best activity in reducing thrombogenicity and the best haemocompatibility. The inclusion of 
PEG segments into the PEG-UAA structure increased its hydrophilicity. The methylene bis(cyclohexyl) segments in Tecoflex PU decreased haemocompatibility. These observations are in good agreement with performed contact angle measurements. The PEG-hexamethylene PU loaded with COC might be a promising material for applications in bioengineering.

Keywords: polymer; cholesteryl oleyl carbonate; platelet; blood compatibility

\section{Introduction}

Polymeric biomaterials are widely used in medical applications [1-3]. Biocompatibility, in particularly blood compatibility (haemocompatibility), is the most important property required for biomedical materials, e.g., stents, implants and devices for hemodialysis, so many researches have tried to improve the blood compatibility of biomaterials [4]. One method to improve the biocompatibility of materials is to cover a material surface with a polymer without altering the bulk physical and mechanical properties [5]. Other attempts have explored the possibility by obtaining new or better properties from a combination of biopolymers and synthetic polymers [6]. For new materials, a key evaluation point is to test their ability to prevent thrombus formation initiated by platelet adhesion in normal and pathologic states. Other parameters like plasma coagulation (e.g., fibrinogen), ADP, thromboxane A2 are involved in thrombus formation during blood coagulation. As the first step in blood coagulation, the initiation of platelet adhesion is important. The so-formed activated platelets accelerate thrombus formation through several actions: (1) secretion of bulk phase agonists (e.g., ADP, thromboxane A2) which attract more platelet adhesion; (2) glycoprotein IIa/IIIb of platelets bind to fibrinogen causing platelet-platelet aggregation and (3) in cooperation with activation of prothrombin and fibrinogen, polymerization of fibrin finally forms hemostatic clots [7].

Polyurethanes, commonly abbreviated PU, owe their names to the urethane (-NH-COO-) units present in the links of connecting polymeric structures. In general polyurethane polymers can be prepared by using a diisocyanate monomer in combination with another molecule containing at least two $\mathrm{OH}$ groups. Polyurethanes have excellent biocompatibility and mechanical flexibility properties [8]. Therefore, starting from 1970s, many applications of urethane-based materials have been reported $[9,10]$.

Liquid crystals are a state of matter that has properties of both solids and liquids. Embedding of liquid crystals has been used in medical applications such as in thermo-responsive systems [11] or in improving gas separation properties of polymeric membranes [12]. Similarly, several surfactants with liquid crystal properties were used for prolonged drug delivery [13]. The liquid crystal cholesteryl oleyl carbonate (COC), has the thickness of the length of phospholipid molecules. Due to its phase transition properties (solid to cholesteric phase), it can behave like a cell membrane [14]. This quality of COC is responsible for its biocompatible and temperature-controllable behavior in biomaterials [14,15].

In this study, two urethane-based polymers, poly(hexamethylene-urethane) (expressed as "hexamethylene PU") and PEG-poly(hexamethylene-urethane) ("PEG-hexamethylene PU") were prepared from new chemical entities (UAA and PEG-UAA). A liquid crystal was embedded into polymeric membranes to obtain new polymer-COC composites. The effects of these polymers and polymer-COC composites on platelet adsorption, platelet activation and $\mathrm{RBC}$-hemolytic potential were evaluated. 
The effects of incorporation of aliphatic and PEG segments into the material on behavior were compared and discussed.

\section{Results and Discussion}

\subsection{Results}

\subsubsection{Characterization of UAA and PEG-UAA}

The identity of UAA and PEG-UAA was determined by FT-IR and NMR (Figures 1 and 2). In the infrared spectra, these polymer-blocks all show a peak around $3300 \mathrm{~cm}^{-1}$ for the urethane $-\mathrm{NH}-$ stretching.

Figure 1. The infrared spectra analysis of (a) UAA; (b) PEG-UAA.

(a)
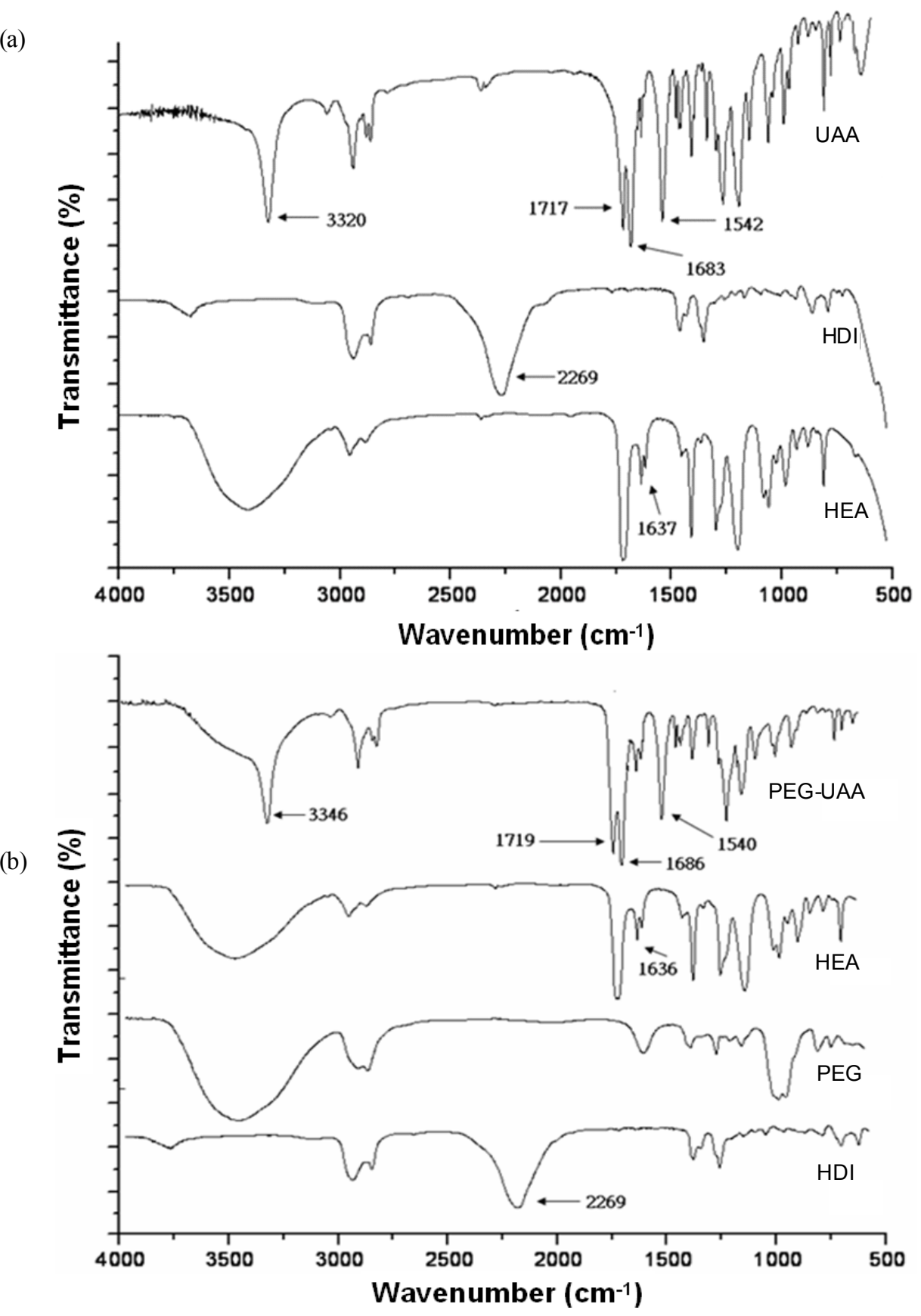
Peaks at 2,930 and 2,855 $\mathrm{cm}^{-1}$ are from $-\mathrm{CH}_{2}-$ symmetric and asymmetric stretching. Peaks at 1,717 or $1,719 \mathrm{~cm}^{-1}$ are from the $-\mathrm{C}=\mathrm{O}$ carbonyl stretching of urethane and peaks around 1,542 or $1,540 \mathrm{~cm}^{-1}$ are due to $-\mathrm{NHCOO}-$ amide $\mathrm{N}-\mathrm{H}$ bending. Peaks at 1,683 or $1,686 \mathrm{~cm}^{-1}$ are from $-\mathrm{C}=\mathrm{O}$ carbonyl stretching of amide. The disappearance of the absorption at 2,269 $\mathrm{cm}^{-1}$ (-NCO-, HDI) and a decrease of the absorption at $1,637 \mathrm{~cm}^{-1}(-\mathrm{C}=\mathrm{C}-$, HEA $)$ indicate a formation of PU with acrylate at both ends of the UAA and PEG-UAA structures (Figure 1). The ${ }^{1} \mathrm{H}-\mathrm{NMR}$ spectra (Figure 2) also confirm the structures of UAA and PEG-UAA, where a difference due to the PEG signal (3.61 ppm) is evident.

Figure 2. ${ }^{1} \mathrm{H}-\mathrm{NMR}$ analysis of (a) UAA; (b) PEH-UAA.

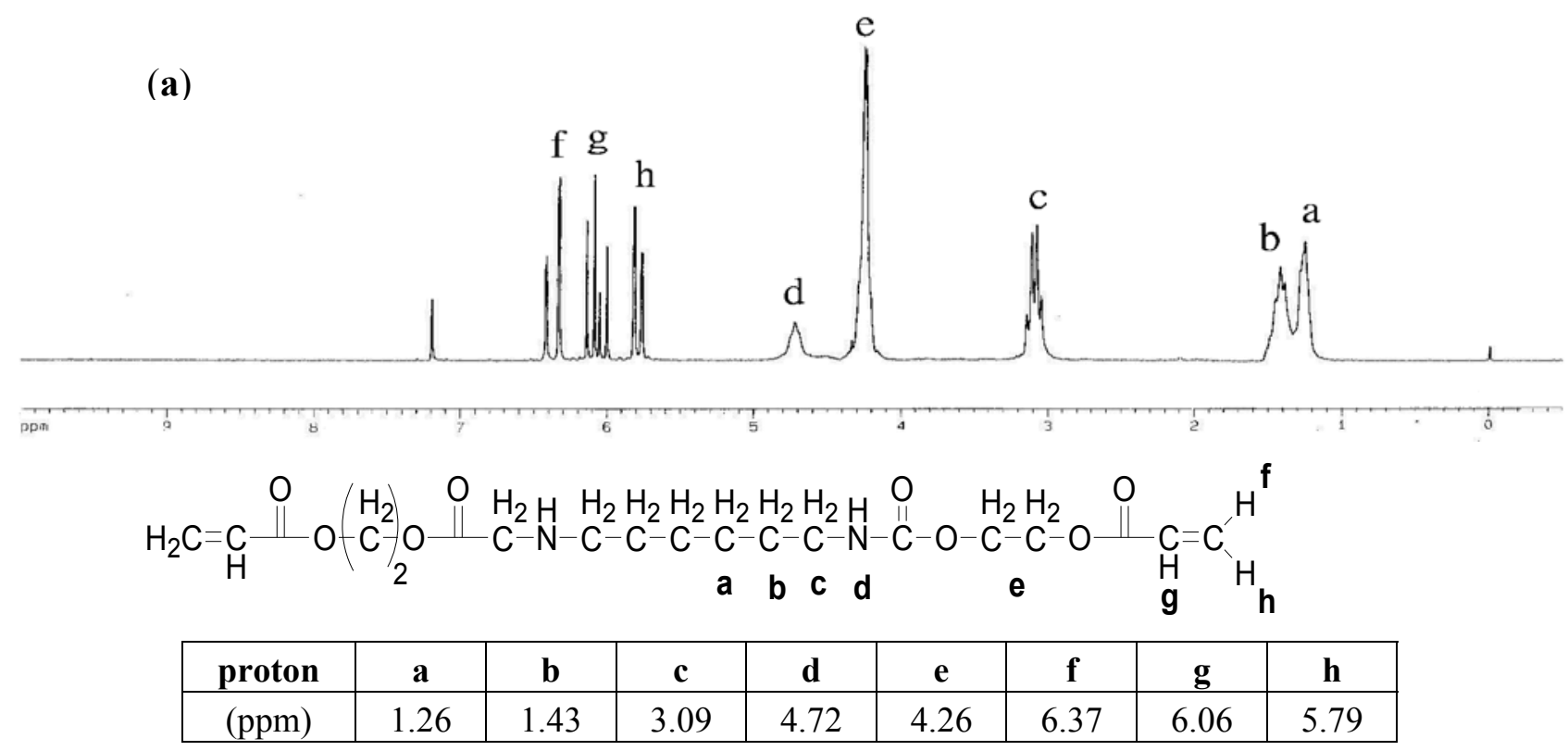

(b)

\section{$\mathrm{k}$}

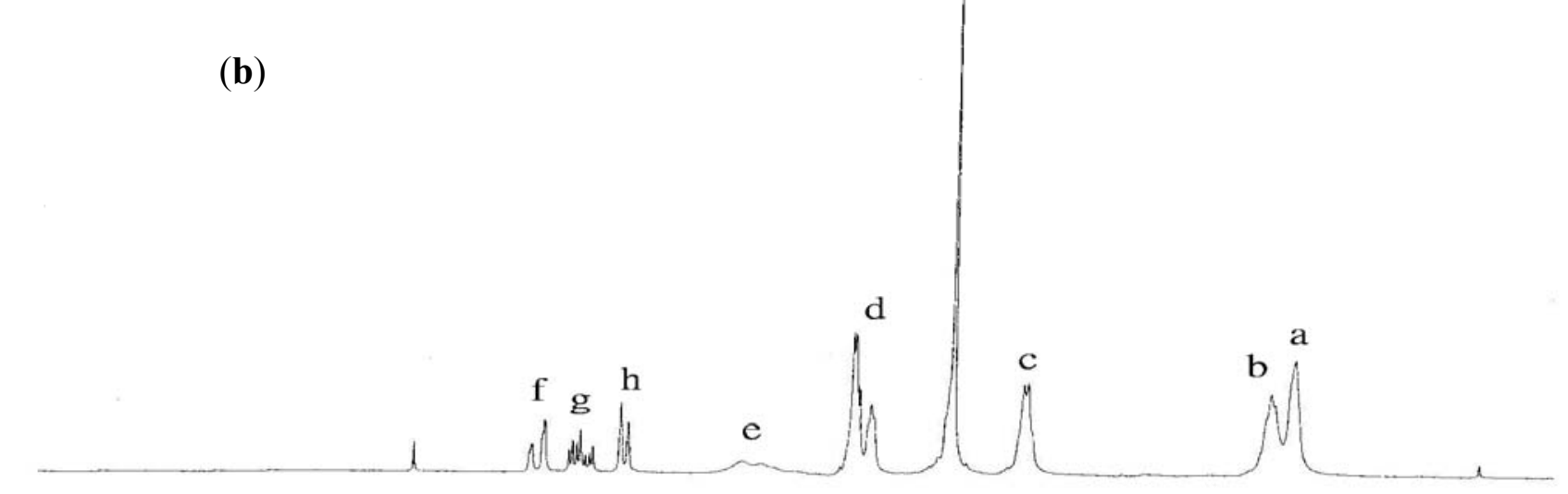

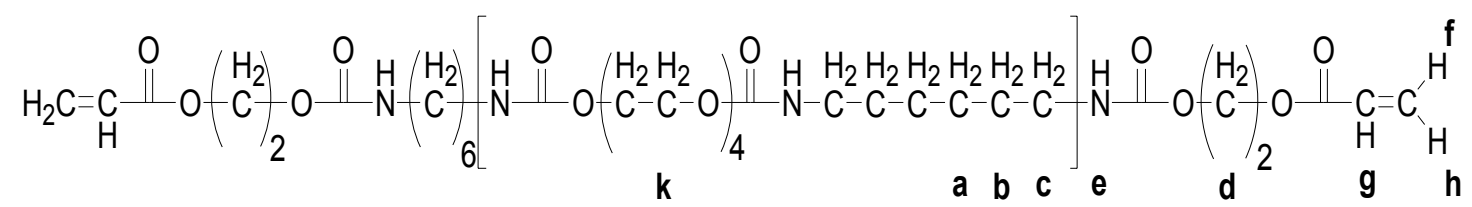

\begin{tabular}{|c|c|c|c|c|c|c|c|c|c|}
\hline proton & a & b & c & d & e & f & g & h & k \\
\hline$(\mathrm{ppm})$ & 1.25 & 1.4 & 3.07 & $4.19 \sim 4.23$ & 4.93 & 6.36 & 6.08 & 5.79 & 3.61 \\
\hline
\end{tabular}


2.1.2. Observation of Surface Morphology and Platelet Adhesion on the Polymers Hexamethylene PU and PEG-Hexamethylene PU and Their COC Composites

Platelet adhesion onto hexamethylene PU and PEG-hexamethylene PU was examined on SEM micrographs (Figures 3a-i). In Figure 3g, the platelets on PMMA show spreading with hyaloplasms and prominent pseudopodial formation (stage IV-V, Cooper's classification, see Table 1) [16]. The platelets on hexamethylene PU (Figure 3a) and PEG-hexamethylene PU (Figure 3d) are in stage II, which describes dendritic and rounded platelets with early pseudopodial formation.

Figure 3. SEM micrographs of platelet adhesion on the surface of hexamethylene PU (a-c) and PEG-hexamethylene PU (d-f) and their COC composites after $60 \mathrm{~min}(2000 \times)$.

(a) Hexamethylene PIJ

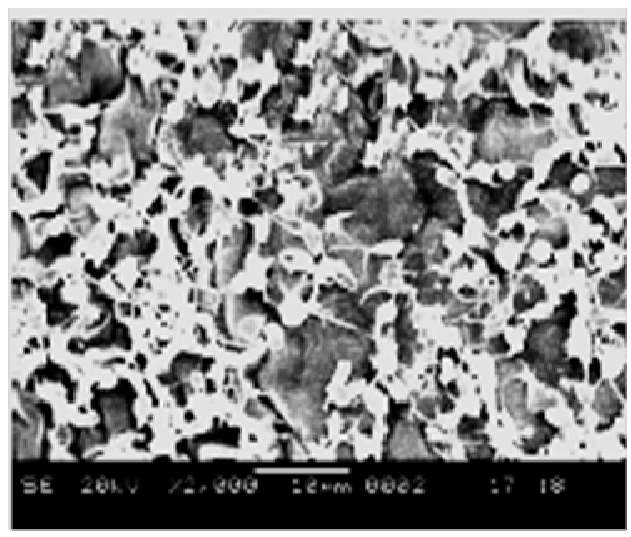

(b) Hexamethylene PU $+\mathrm{COC}\left(5^{\circ}\right)$

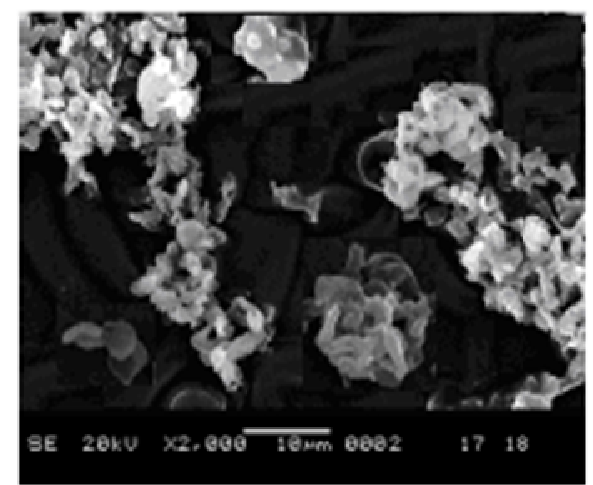

(c) Hexamethylene PU $+\mathrm{COC}\left(10^{\circ} \mathrm{b}\right)$

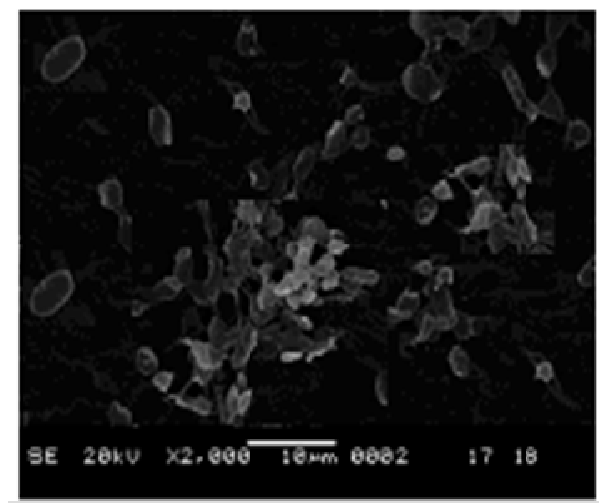

(d) PEG-hexamethylene PU

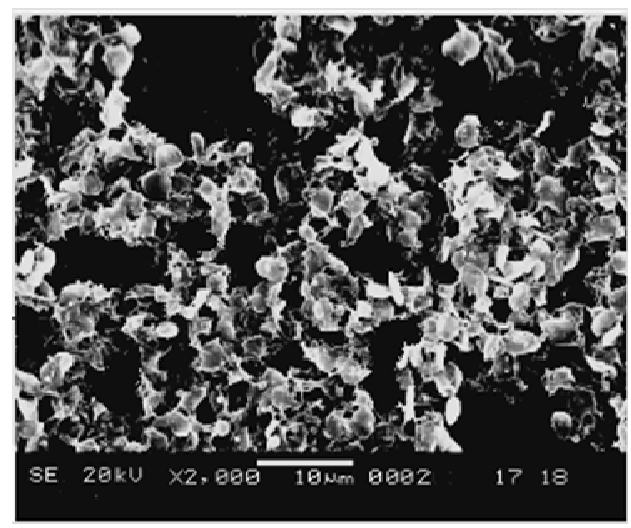

(e) PEG-hexamethylene PU $+\mathrm{COC}\left(5^{\circ}\right)$

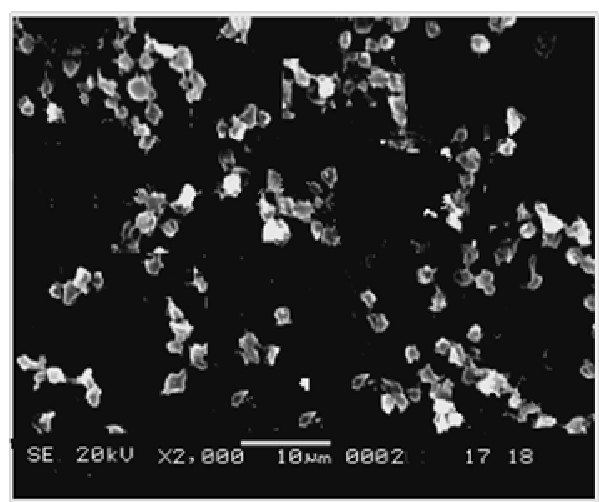

(f) PEG-hexamethylene PU +COC (10

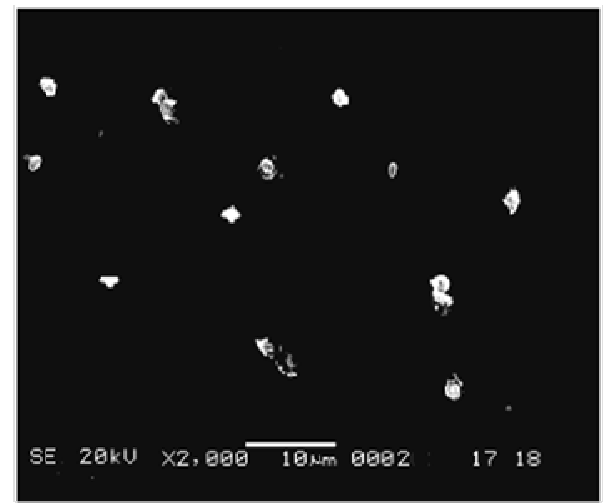


Figure 3. Cont.

(g) Control: PMMA

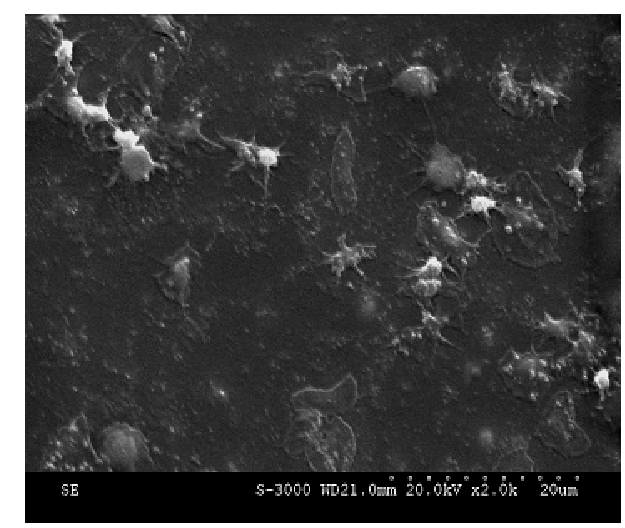

(i) Control: Tecoflex PU+ COC $\left(10^{\circ}\right.$ is)

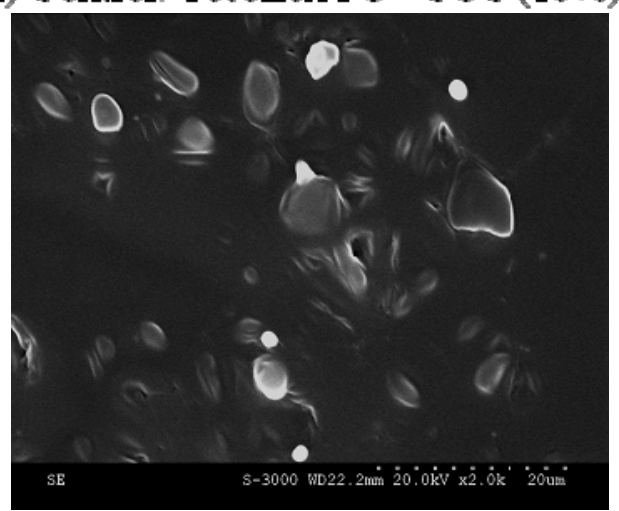

(h) Control: PMMA+ $\mathrm{COC}\left(10^{\circ} \mathrm{i}\right)$

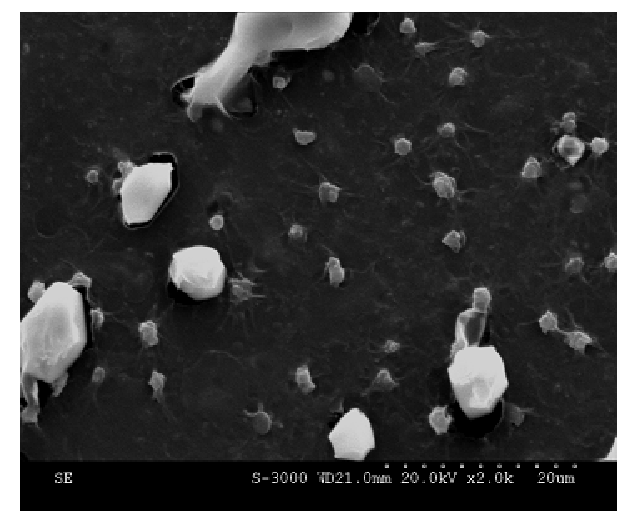

After COC was embedded into the polymers, the extent of platelet adhesion decreased proportional with COC content (Figures 3b-f). Only rounded platelets with no pseudopodia (stage I) were observed on PEG-hexamethylene + COC (10\%), suggesting minimal platelet adsorption and activation (Figure 3f). The percentages of COC embedded in the polymers of up to $10 \%$ did not show changes in their tensile strength $(\sim 5 \mathrm{MPa})$ and surface morphology. The low platelet adhesion onto the PEG-hexamethylene PU + COC (10\%) indicates a great improvement compared to other PU materials for haemocompatibility.

\subsubsection{Contact Angle Measurements of Polymers and Polymer-COC Composites}

The contact angles for various polymer and polymer-COC composites were measured to compare the hydrophilicity of materials under investigation (Table 1). The water contact angle of the original polymers (PMMA, Tecoflex PU, hexamethylene PU and PEG-hexamethylene PU) was decreased after incorporation of COC (10\%). The largest differences in contact angle after COC incorporation were found in PMMA, hexamethylene PU and PEG-hexamethylene PU.

Table 1. The contact angle on polymer and polymer-COC composites.

\begin{tabular}{ccccc}
\hline Contact angle (degree) & PMMA & Tecoflex PU & Hexamethylene PU & PEG-hexamethylene PU \\
\hline Polymer & $95 \pm 0.5$ & $89 \pm 0.3$ & $79 \pm 0.4$ & $69 \pm 0.4$ \\
Polymer + 10\% COC & $80 \pm 0.8$ & $84 \pm 1.1$ & $70 \pm 0.9$ & $58 \pm 0.6$ \\
\hline
\end{tabular}




\subsubsection{Functional Assay for Evaluation of Platelet Activation: P-Selectin Measurements}

P-selectin is a surface-component of platelet granules that appears when platelets are stimulated and activated [17]. P-selectin is therefore directly correlated to activated platelet count [18]. To quantify the antithrombotic effect mediated by activated platelets, P-selectin measurements were performed. In Figure 4, the production of P-selectin from samples in PRP blood is demonstrated. PRP blood incubated with PMMA resulted in extensive quantities of P-selectin. This suggests substantial platelet activation and indicates that PMMA has poor blood compatibility. P-selectin produced by Tecoflex PU was 50\% less and PEG-hexamethylene showed a P-selectin value close to the negative control. The polymers with embedded COC decreased the production of P-selectin even more and showed that inclusion of COC improves haemocompatibility of the material to human blood.

Figure 4. The effects of COC embedded in polymer composites on P-selectin levels. * $\mathrm{P}<0.05$ for hexamethylene PU + COC (10\%) and PEG-hexamethylene PU + COC $(10 \%)$ compared to their non-COC groups.

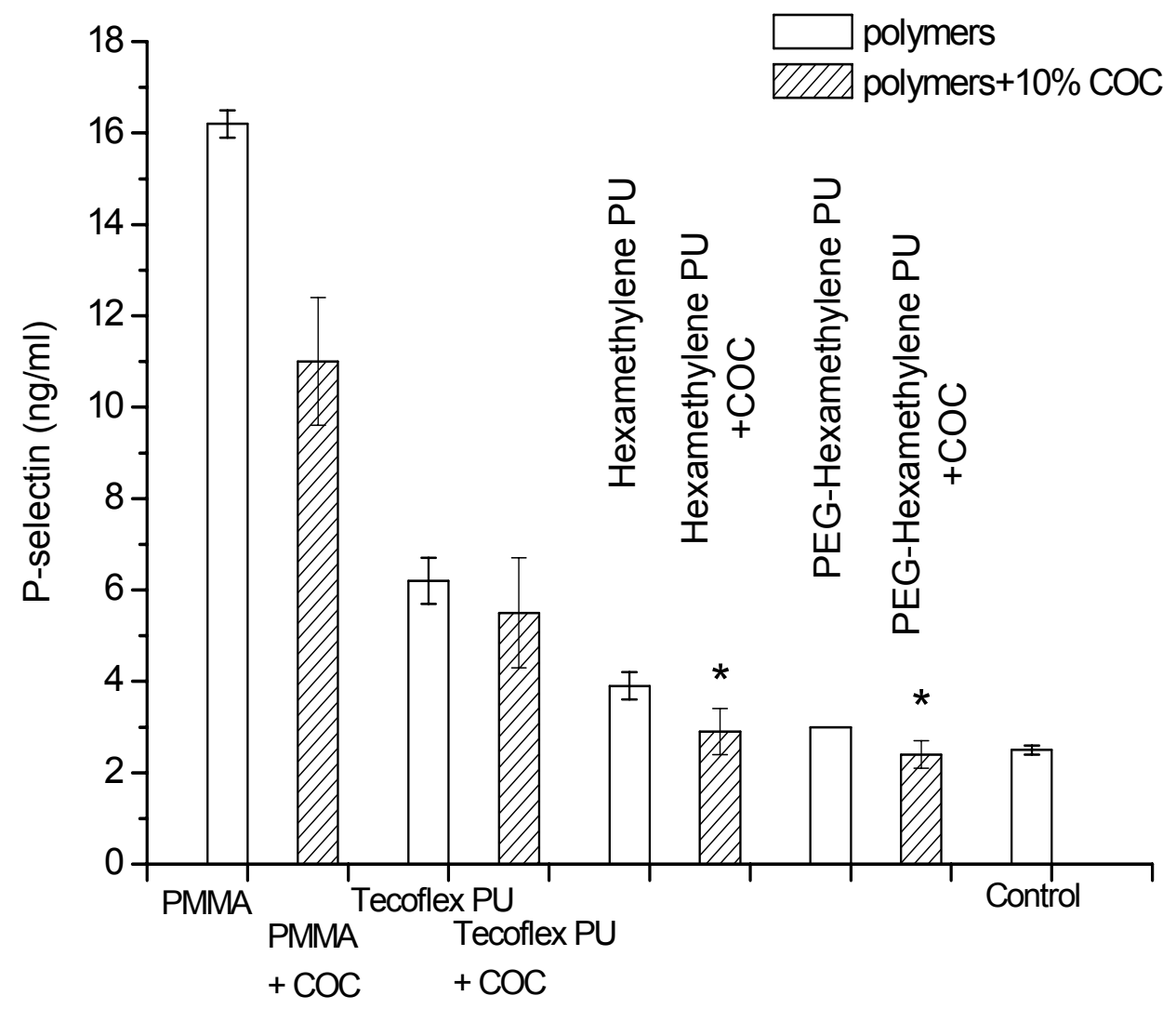

2.1.4. Blood Clotting Properties of Hexamethylene PU and PEG-Hexamethylene PU and Their COC Composites

Spreading and flattening of platelets into irregular shapes on materials indicates activation of the platelets. These activated platelets trigger thrombus formation which indicates that the material is less compatible with human blood [19]. The activity in reducing thrombogenicity of materials is quantitatively expressed by a relative parameter known as blood clotting index (BCI). A larger BCI value ( $=$ a tendency toward less coagulation) means an increase in haemocompatibility. Figure 5 shows 
the tendency of blood coagulation induced by the polymers and the influence of embedded COC content on the BCI values. An increase in the BCI value was found for polymers with higher COC content (from 0 to $10 \%$ ). In accordance with the finding on platelet adhesion, the addition of COC into the polymers improves the biocompatibility with human blood. PEG-hexamethylene PU $+10 \% \mathrm{COC}$ triggered the least blood coagulation and showed the highest BCI value. The BCI-test indicates the following order in haemocompatibility of the polymers (with or without COC): PEG-hexamethylene $>$ hexamethylene PU > Tecoflex PU > PMMA.

Figure 5. Effects of COC content embedded in polymer composites on blood clotting index $(\mathrm{BCI}) .{ }^{*} \mathrm{P}<0.05$ compared to the non-COC group.

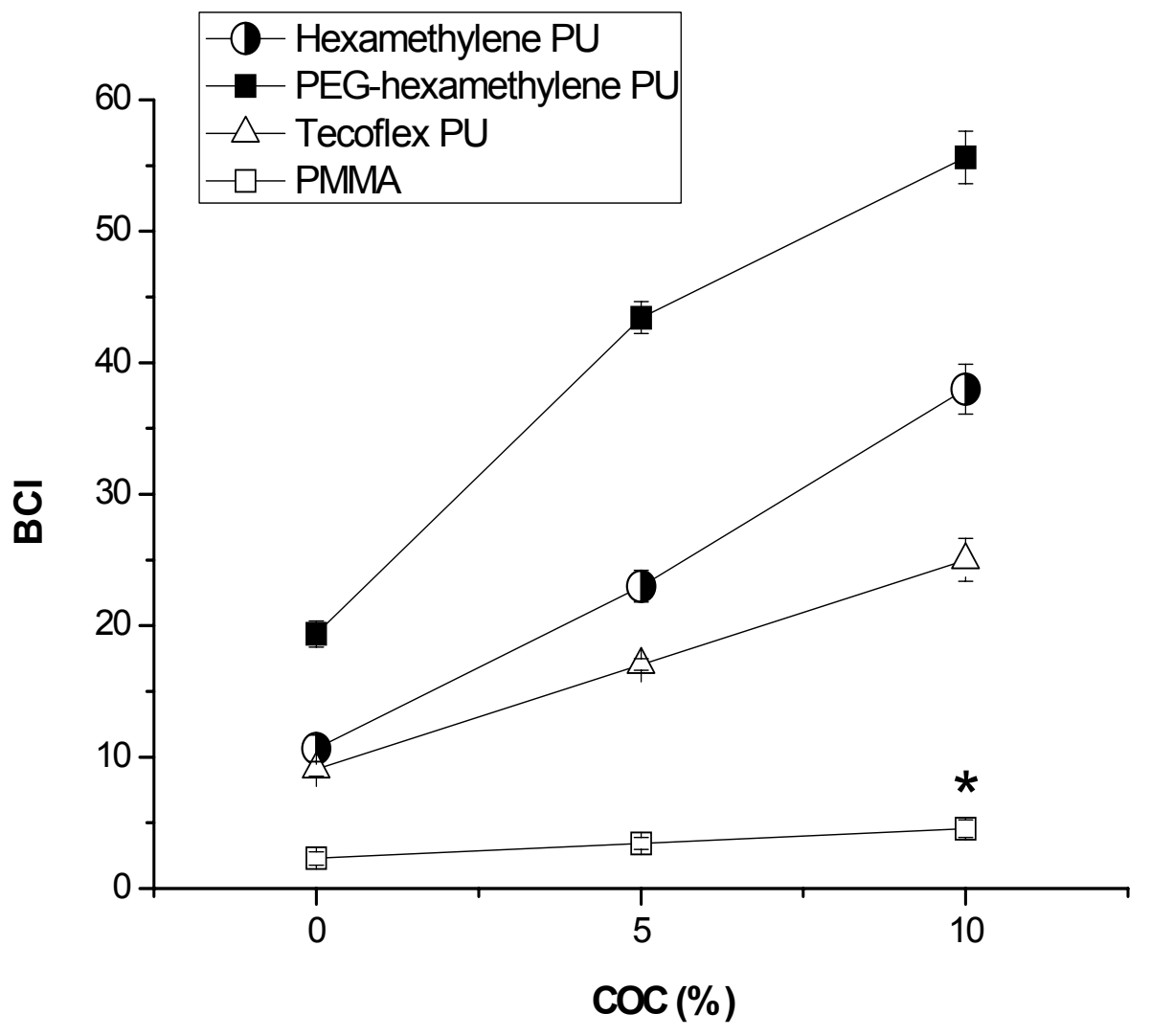

\subsubsection{The Hemolysis Properties of the Polymers and Polymer-COC Composites}

Good biocompatibility to human blood requires not only limited platelet adhesion and activation, but also less hemolysis of red blood cells (RBC). The hemolysis ratio (HR) expresses the extent of degraded $\mathrm{RBC}$ after a material in contact with blood.

A lower value of HR means less hemolysis and a better blood compatibility. It is known from the literature that the HR value for biomaterials used in medical applications should be below 5\% [20]. In Figure 6, the HR values of the polymers decrease in the order: PMMA $>>$ Tecoflex PU $>$ hexa-methylene $\mathrm{PU}=$ PEG-hexamethylene PU. Inclusion of COC in the polymers further decreases their HR values. Again, the PEG-hexamethylene PU $+10 \%$ COC showed the best biocompatibility with almost no hemolysis. 
Figure 6. Effects of COC content embedded in polymer composites on hemolysis ratio (HR) values.

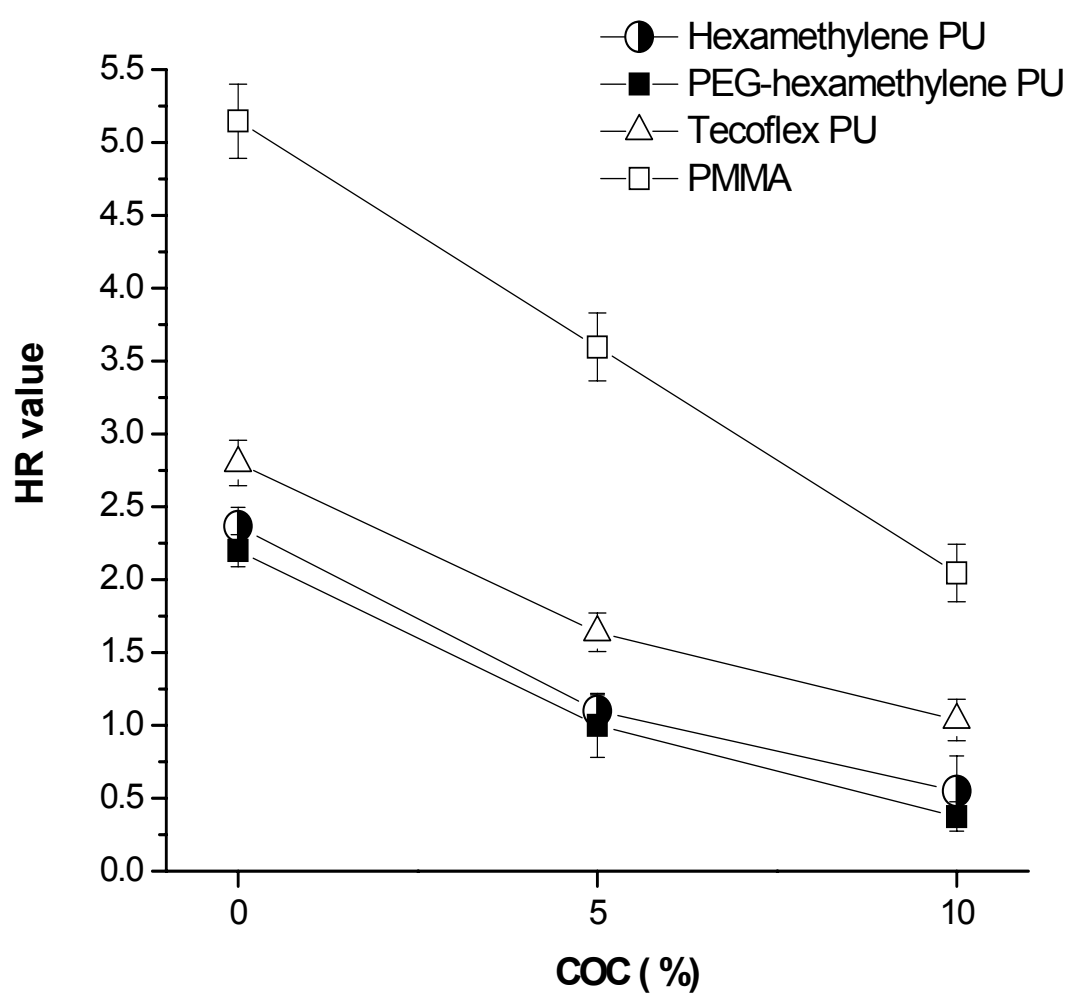

\subsection{Discussion}

PEG is used extensively in biomaterials to impart low protein and cell adsorption on surfaces [21] and to reduce immunogenicity and to increase the resistance of proteolytic cleavage in drug targeting [22]. PEG modification improves the hydrophilicity of materials. However, the mechanical strength of materials could be reduced after addition of PEG. In our study, short PEG segments were incorporated into hexamethylene PU and its tensile strength did not changed (data not shown). Furthermore, the increased hydrophilicity of PEG-hexamethylene PU by inclusion of COC decreases platelet adhesion substantially.

Zhang et al. and Yuan et al. studied polyurethane hydrophilicity with surface-pendent ammonium zwitterions [23,24]. A more hydrophilic surface resulted in less platelet adhesion and better heamocompatility. However, sometimes the opposite results regarding hydrophilicity in connection to haemocompatibility were observed [25,26] (e.g., incorporation of $\mathrm{COOH}$ as pendent groups in a polyurethane anionomer showed increased platelet adhesion even though hydrophilicity was increased). In our study, the bis-cyclohexyl structure (in Tecoflex PU) is less hydrophilic than the hexamethylene group (in hexamethylene PU), and the PEG-hexamethylene structure is the most hydrophilic. The reduction of platelet adhesion and contact angles is in good agreement with the hydrophilicity of the polymers (Table 1).

When the surface chemistry of materials changes, it might alter the adsorption of fibrinogen [27]. It is also known that the thrombogenicity of materials is related to their adsorbed fibrinogen. We measured platelet adhesion because this process is mediated by plasma protein adsorption, especially the adsorbed fibrinogen and von Willebrand factor [28]. Therefore, the platelet adhesion can be an 
indication for fibrinogen adsorption and formation of thrombi (thrombogenicity). A grafted phospholipid analogous on a PU surface showed an improvement in platelet adhesion [29-31]. The presence of COC molecules acts like phospholipid moieties of plasma membranes and therefore shows better haemocompatibility. Upon activation of platelets, cell shape change, platelet-platelet contact and platelet adhesion are promoted leading to the release of their intracellular granular contents (e.g., P-selectin) [32]. Therefore, By means of reducing platelet adhesion or inactivating platelets (e.g., shielding platelet GP IIb/IIIa receptor), thrombotic process could be retarded [33]. The thrombogenicity of materials was measured quantitatively by the release of P-selectin and showed less activation of platelets on polymer-COC composites.

We also showed a modification of surface chemistry affected blood coagulation (BCI). The inclusion of COC lowers blood coagulation (with an increased of BCI values) so less thrombogenicity occurred. This is probably due to a reduction of platelet activation (less P-selectin production, Figure 4) and an increase of the hydrophilicity (Table 1) and less platelet adhesion (Figure 3).

RBC hemolysis determines the haemocompatibility of a material regarding its potential on degrading red blood cells. The COC in the polymer composites was in an isotropic-cholesteric state above its phase transition temperature $\left(\sim 20^{\circ} \mathrm{C}\right)$. This characteristic might contribute to a lower RBC hemolysis of the polymer-COC composites (Figures 6).

\section{Experimental Section}

\subsection{General}

Perkin-Elmer-824 and Bruker NMR $(200 \mathrm{MHz})$ were used to obtain IR and NMR spectra. A commercial PU (Tecoflex; CAS registry number 76600-67-4), 2-hydroxyethyl acrylate (HEA), 1,6-diisocyanatohexane (HDI) and polyethylene glycol (PEG) $\left(\mathrm{HO}\left[\mathrm{CH}_{2} \mathrm{CH}_{2} \mathrm{O}\right]_{4} \mathrm{H}, \mathrm{Mw}=200\right)$ were purchased from Fluka (Buchs, Switzerland). Cholesteryl oleyl carbonate (COC) was obtained from Aldrich (Milwaukee, WI, USA). 1-Hydroxycyclohexylphenyl ketone (HCPK) was from Ciba-Geigy (Basel, Switzerland). Other chemicals and reagents used were of analytical grade. The chemical structures of the two designed polymer-blocks are shown in Scheme 1.

Scheme 1. The structures of (a) UAA; (b) PEG-UAA; and their PU polymers compared to (c) Tecoflex PU.

(a) $\underline{\text { UAA }}$<smiles>C=CC(=O)OCCCC(C)(C)NC(=O)OC(C)(C)NC(=O)OCC(C)(C)C</smiles>

Radical polymerization Hexamethylene PU

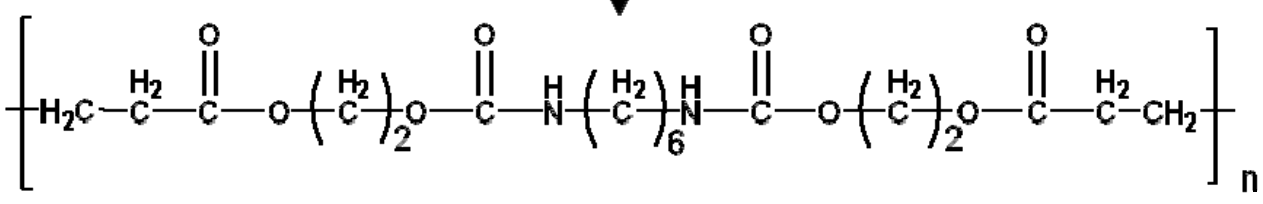


Scheme 1. Cont.

(b) PEG-UAA

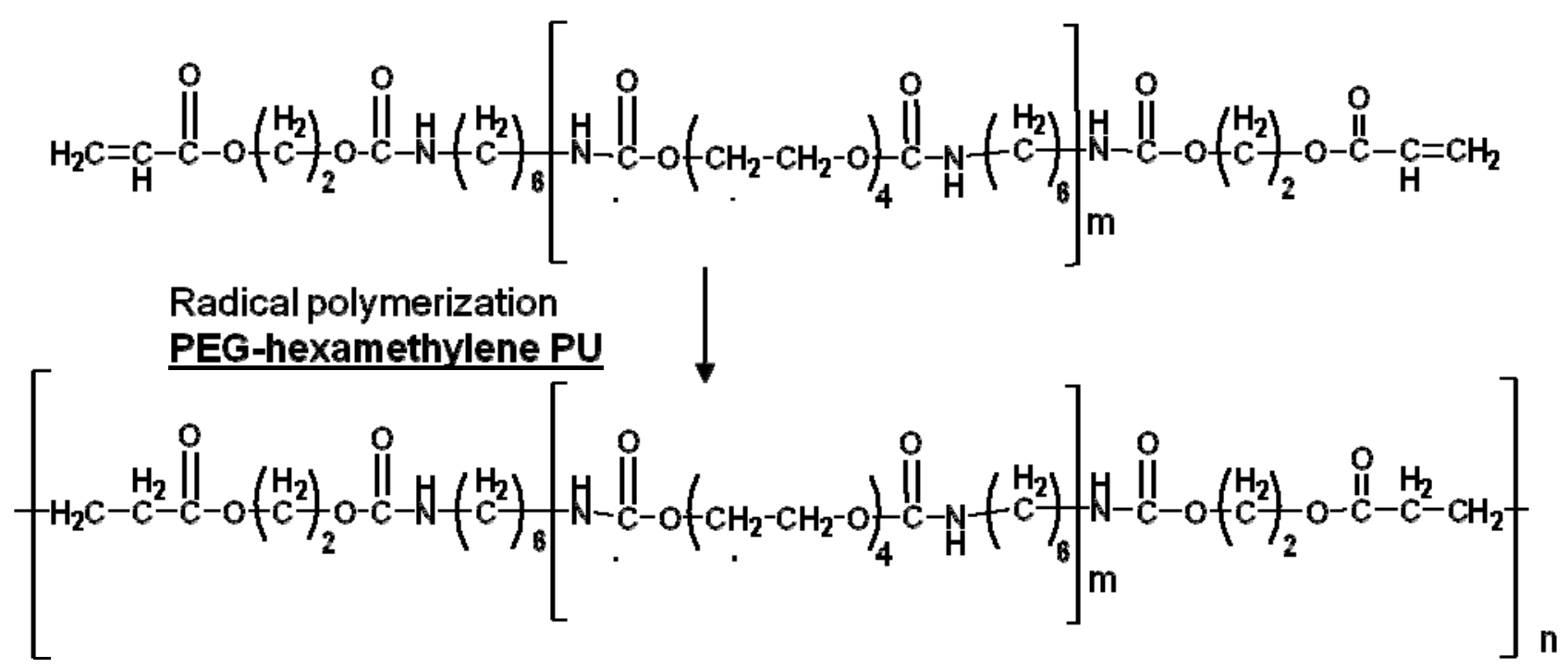

(c) Tecoflex PU

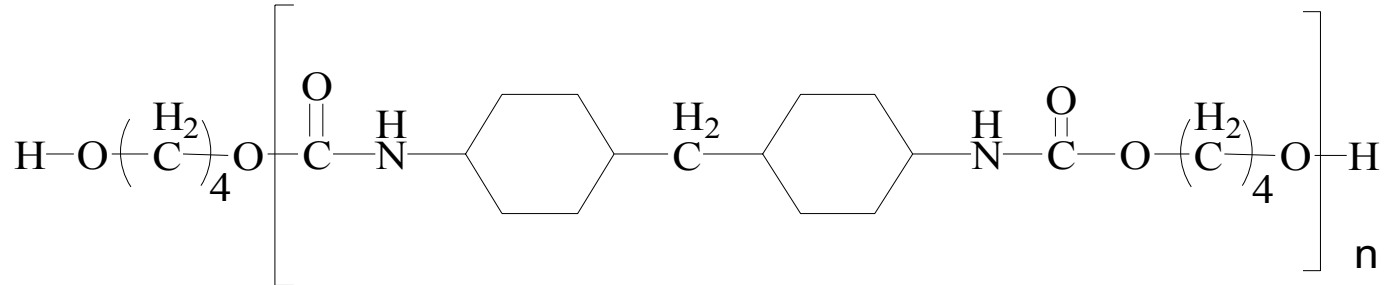

\subsection{Synthesis of UAA and PEG-UAA as Polymer-Blocks}

UAA was synthesized via a one-step process as shown in Scheme 2. 1,6-Diisocyanatohexane (HDI) (1) and 2-hydroxyethyl acrylate (HEA) (2) with a $\mathrm{NCO} / \mathrm{OH}$ molar ratio of 1:2 were mixed in anhydrous DMF in a three-neck reaction flask under a dry nitrogen purge, heated at $50{ }^{\circ} \mathrm{C}$ and allowed to react for $5 \mathrm{~h}$. The UAA was precipitated and purified in ethyl ether and dried under vacuum.

For PEG-UAA, 1,6-diisocyanatohexane (HDI) (1, $1.218 \mathrm{~g})$ and PEG (3, $0.362 \mathrm{~g})$ with a NCO/OH molar ratio of 4:1 were mixed in anhydrous DMF in a three-neck reaction flask under a dry nitrogen purge, heated at $60{ }^{\circ} \mathrm{C}$ and allowed to react for $5 \mathrm{~h}$. The product 4 was precipitated in anhydrous ethyl ether and vacuum-dried. Then 2-hydroxyethyl acrylate (HEA, 2, $1.682 \mathrm{~g}$ ) was added to react with product 4 at $50{ }^{\circ} \mathrm{C}$ for $5 \mathrm{~h}$. The PEG-UAA was precipitated, purified in ethyl ether and vacuum dried.

\subsection{Preparation of Polymers and Polymer-Liquid Crystal Composites via Photo-Polymerization}

The two polymers, hexamethylene PU and PEG-hexamethylene PU were synthesized from UAA and PEG-UAA, respectively, by photopolymerization of 2-hydroxyethyl acrylate and 1,6-diisocyanatohexane with or without polyethylene glycol. Polymer-liquid crystal composites were prepared in the presence of the liquid crystal (COC) during the polymerization. In detail, acrylic monomers were first mixed with $3 \%$ radical initiator (HCPK). A clear melting solution was formed around $60{ }^{\circ} \mathrm{C}-70{ }^{\circ} \mathrm{C}$ and various amounts of $\mathrm{COC}$ were added to obtain weight ratios from $0 \%$ to $10 \%$. 
Scheme 2. The synthesis of (a) UAA and (b) PEG-UAA.

(a) for UAA

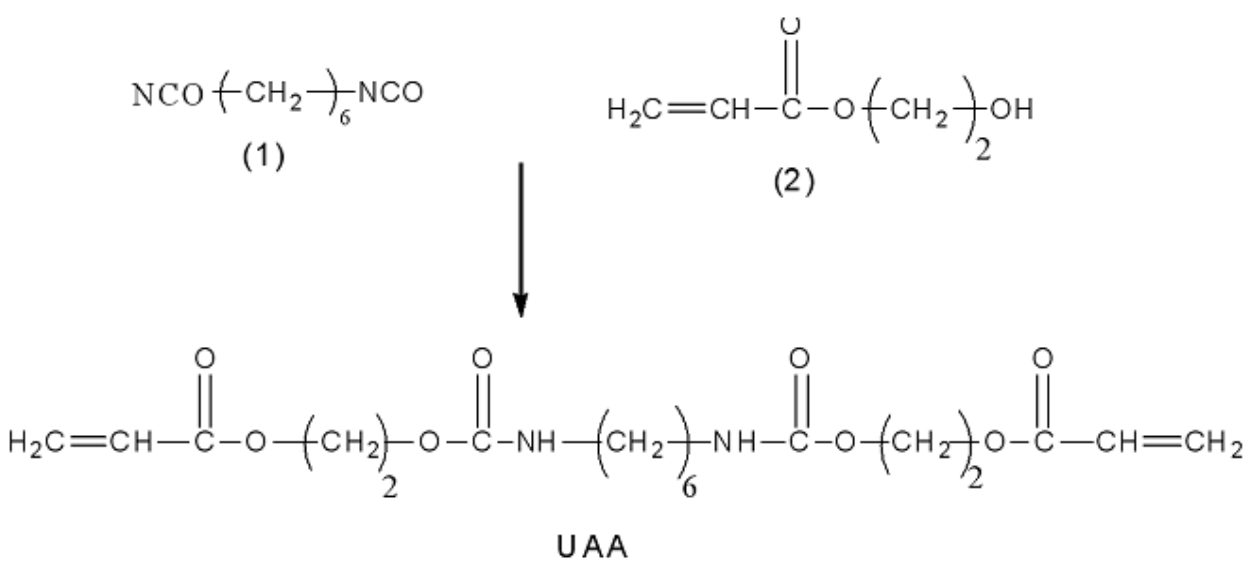

(b) for PEG-UAA

$$
\mathrm{NCO}+\mathrm{CH}_{2}+{ }_{6} \mathrm{NCO} \quad \mathrm{HO}+\mathrm{CH}_{2} \mathrm{CH}_{2} \mathrm{O}+{ }_{4}+\mathrm{H}
$$

(1)

(3)

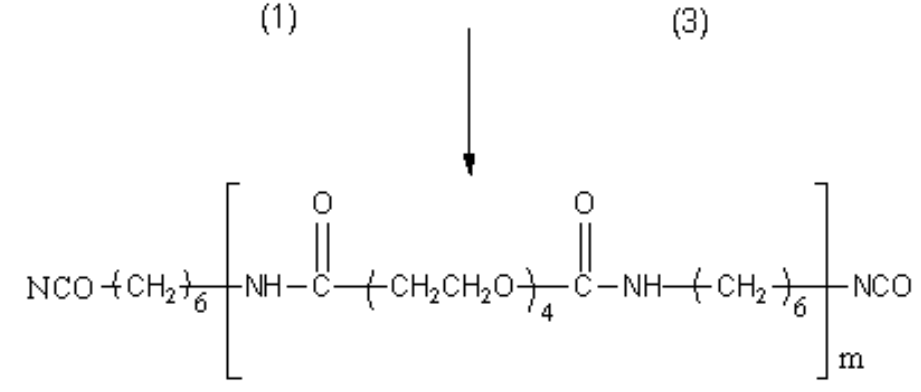

(4)

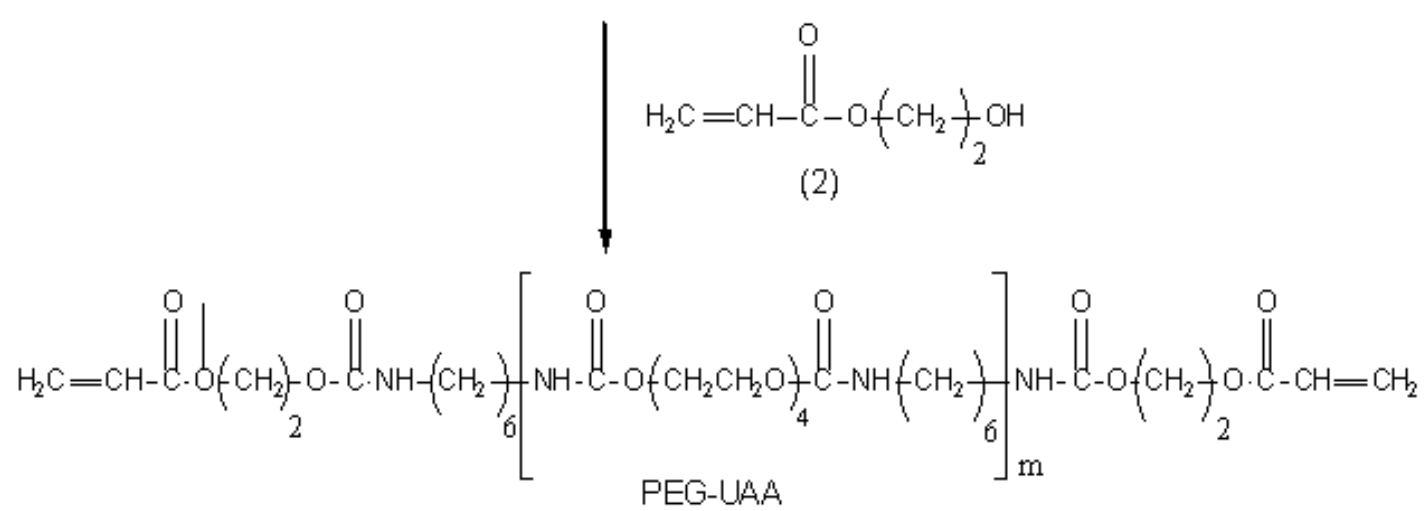

A mold with a hollow spacer $(0.3 \mathrm{~mm}$ in depth) on a bottom lining (polypropylene) was set on a glass plate and then the solution was cast into the mold. After the polypropylene lining cover was placed over the mold, another glass plate was placed and the whole assembly was fixed by clips. An UV-lamp was applied for photo-polymerization during $60 \mathrm{~s}$. After polymerization, the polymer and polymer-COC composites were vacuum dried in a dessicator. 


\subsection{Tensile Strength Analysis and Contact Angle Measurements of Polymers and Polymer-COC Composites}

The tensile strength of the polymers and polymer-COC composites was measured with universal tensile measuring equipment (Instron SSTM-1, Japan) at a crosshead speed of $20 \mathrm{~mm} / \mathrm{min}$. The thickness of the films was $0.3 \mathrm{~mm}$. The contact angle of water was measured at $25{ }^{\circ} \mathrm{C}$ with the sessile drop method (DSA100, Kruss, Germany).

\subsection{Observation of Platelet Adhesion by SEM and Evaluation of Platelet Activation (Coope's Classification)}

Fresh blood from healthy donors (three persons) supplemented with citrate dextrose (ACD; anticoagulant) (9:1) was centrifuged at $100 \times$ for $10 \mathrm{~min}$ at $4{ }^{\circ} \mathrm{C}$ to obtain platelet-rich plasma (PRP). The synthesized materials were rinsed three times with deionized water and then immersed in $3 \mathrm{ml}$ PRP (average platelet number is $5.4 \times 10^{5} \mathrm{~mL}^{-1}$ ) which was preheated at $37^{\circ} \mathrm{C}$. After $1 \mathrm{~h}$ incubation at $37^{\circ} \mathrm{C}$, the materials were washed with PBS to remove non-adherent platelets. The adhered platelets were fixed with $2 \%(\mathrm{w} / \mathrm{v})$ glutaraldehyde/ PBS for $5 \mathrm{~min}$ at $4{ }^{\circ} \mathrm{C}$. After washing with PBS thoroughly, the platelet attached materials were vacuum dried prior to SEM studies. $100 \mathrm{~s}$ were applied to make materials shadowed with Pt-Pd alloy at 15mA. Based on the SEM observations (Hitachi S-3000N), the amount of platelet adhesion and the morphology of adhesive platelets were evaluated according to Cooper's classification (Table 2).

Table 2. Cooper's classification for platelet adsorption.

\begin{tabular}{ll}
\hline Stage & Description \\
\hline I & Rounded platelets with no pseudopodia \\
II & Dendritic, rounded platelets with early pseudopodial formation \\
III & Spreading, dendritic platelets \\
IV & Spreading platelets and their hyaloplasm with prominent pseudopodial formation \\
V & Fully spread platelets over the entire surface \\
\hline
\end{tabular}

\subsection{Evaluation of Platelet Activation by P-Selectin Measurements}

The P-selectin assay employs a quantitative sandwich immunoassay kit (R\&D systems, Inc., MN, USA). A monoclonal antibody specific for P-selectin was pre-coated onto a microplate. Standards, samples and controls were pipetted into microwells and then added with a polyclonal antibody specific for P-selectin which had been conjugated with horseradish peroxidase. After removal of unbound conjugated antibody, a substrate was added and color developed, which is proportional to P-selectin concentration. PRP without incubation of the polymers was used as the control, for comparison. A known concentration of P-selectin $(36.86 \mathrm{ng} / \mathrm{mL})$ included in the kit was measured at $450 \mathrm{~nm}$ as a standard for establishing a standard curve. Experimental procedures were followed as described in the kit brochure. 


\subsection{In Vitro Blood Compatibility Test: Blood Clotting Measurements}

The number of RBC originates from healthy donors and from different experimental days was checked colorimetrically (absorbance of hemoglobin at $542 \mathrm{~nm}$ ) and adjusted to the same value with PBS. The synthesized materials were placed into flat-bottom bottles. These bottles were placed in a thermostatic water bath at $37{ }^{\circ} \mathrm{C}$ for $5 \mathrm{~min}$. Blood $(0.27 \mathrm{~mL})$ was taken from ACD-whole blood $(0.3 \mathrm{~mL})$ plus $\mathrm{CaCl}_{2}(0.2 \mathrm{~mol} / \mathrm{L}, 0.024 \mathrm{~mL})$ and slowly dropped on the surface of the materials, until the material was completely covered. The bottles were further incubated in an incubator at $37{ }^{\circ} \mathrm{C}$. After $10 \mathrm{~min}$, deionized water $(10 \mathrm{~mL})$ was carefully added without disturbing the clotted blood. Subsequently, an aliquot $(10 \mathrm{~mL})$ from each bottle were taken and centrifuged at $100 \times \mathrm{g}$ for $30 \mathrm{~s}$. The supernatant was decanted into a tube and made up with deionized water to $50 \mathrm{~mL}$ (kept in $37^{\circ} \mathrm{C}$ ) and held for $60 \mathrm{~min}$. The blood clotting test was carried out by measuring the relative absorbance of blood in the supernatant at $542 \mathrm{~nm}$. The control was taken to measure the absorbance of the ACD-whole blood (without $\mathrm{CaCl}_{2}$ ) diluted with deionized water (to $50 \mathrm{~mL}$ ) in glass vials. An increase of the relative blood clotting index $(\mathrm{BCI})$ indicates less blood clotting occurred because more $\mathrm{RBC}$ remained in the supernatant. The relative BCI can be calculated by an equation below:

Relative $\mathrm{BCI}=100 \times\left(\mathrm{Abs} 542 \mathrm{~nm}\right.$ of supernatant from $\mathrm{ACD}$ whole blood $+\mathrm{CaCl}_{2}$ with a material $) /$

(Total hemoglobin in ACD whole blood)

\subsection{In Vitro Blood Compatibility Test: Hemolysis Ratio Measurements}

The materials were rinsed three times with deionized water and normal saline before being transferred and placed into flat-bottom bottles. Normal saline $(10 \mathrm{~mL})$ was added to the bottles and kept at $37{ }^{\circ} \mathrm{C}$ in a shaking water bath (shaking rate $=100$ times per hour). After 60 min, diluted ACDwhole blood ( $8 \mathrm{~mL}$ ACD-whole blood diluted with $10 \mathrm{~mL}$ normal saline, $0.2 \mathrm{~mL}$ ) was dropped into the bottles. The materials were incubated with the blood solution for another $60 \mathrm{~min}$. Next, the blood solution were aspirated and centrifuged at $100 \times \mathrm{g}$ for $5 \mathrm{~min}$. The obtained supernatant was measured at the absorbance of $542 \mathrm{~nm}$ (absorbance of hemoglobin) by a spectrophotometer (Metertech SP8001, Taiwan). The hemolysis ratio (HR) was obtained by the equation: $\mathrm{HR}=100 \times \underline{(\mathrm{AS}-\mathrm{AN})} / \underline{(\mathrm{AP}-\mathrm{AN})}$. where AS is the absorbance of obtained supernatants after in contact with samples. AP and AN denote the absorbance of the positive control $(0.2 \mathrm{~mL}$ diluted ACD-whole blood $+10 \mathrm{~mL}$ deionized water), and the negative control $(0.2 \mathrm{~mL}$ diluted ACD-whole blood $+10 \mathrm{~mL}$ normal saline), respectively. Therefore, (AP-AN) denotes a total hemolysis.

\subsection{Statistical Analysis}

Data from each group $(n \geq 6)$ in different experimental days were analyzed for P-selectin and blood clotting index. A two-tailed student's unpaired test was used to compare the mean values of two populations of continuous data. Statistical analyses were based on Student's $t$-test using Prism software (GraphPAD Inc., San Diego, CA, USA). 


\section{Conclusions}

In this study, the synthesized polymers hexamethylene PU (from UAA) and PEG-hexamethylene PU (from PEG-UAA) were evaluated for their haemocompatibility. These polymers demonstrated better haemocompatibility than a commercial PU (Tecoflex). Inclusion of COC (10\%) into PEG-hexamethylene polymer showed even better blood compatibility because of the decreased platelet adhesion and activation, lowering the induction of blood coagulation and hemolysis. The findings indicate that modified PU-materials show promising potential for biomedical applications.

\section{Conflict of Interest}

The authors declare no conflict of interest.

\section{References}

1. Ravi, S.; Qu, Z.; Chaikof, E.L. Polymeric materials for tissue engineering of arterial substitutes. Vascular 2009, 17, S45-S54.

2. Sahithi, K.; Swetha, M.; Ramasamy, K.; Srinivasan, N.; Selvamurugan, N. Polymeric composites containing carbon nanotubes for bone tissue engineering. Int. J. Biol. Macromol. 2010, 46, 281-283.

3. Rosellini, E.; Cristallini, C.; Barbani, N.; Vozzi, G.; D’Acunto, M.; Ciardelli, G.; Giusti, P. New bioartificial systems and biodegradable synthetic polymers for cardiac tissue engineering: A preliminary screening. Biomed. Eng.-Appl. Basis Commun. 2010, 22, 497-507.

4. Guo, J.; Feng, Y.; Ye, Y.; Zhao, H. Construction of hemocompatible polycarbonate urethane with sulfoammonium zwitterionic polyethylene glycol. J. Appl. Polym. Sci. 2011, 122, 1084-1091.

5. Wang, Y.; Xu, W.; Chen, Y. Surface modification on polyurethanes by using bioactive carboxymethylated fungal glucan from Poria cocos. Colloid Surf. B-Bisointerfaces 2010, 81, 629-633.

6. Shih, M.F.; Shau, M.D.; Chang, M.Y.; Chiou, S.K.; Chang, J.K.; Cherng, J.Y. Platelet adsorption and hemolytic properties of liquid crystal/composite polymers. Int. J. Pharm. 2006, 327, 117-125.

7. Grunkemeier, J.M.; Tsai, W.B.; Horbett, T.A. Hemocompatibility of treated polystyrene substrates: contact activation, platelet adhesion, and procoagulant activity of adherent platelets. J. Biomed. Mater. Res. 1998, 41, 657-670.

8. Rangwala, H.S.; Ionita, C.N.; Rudin, S.; Baier, R.E. Partially polyurethane-covered stent for cerebral aneurysm treatment. J. Biomed. Mater. Res. Part B 2009, 89, 415-429.

9. McBane, J.E.; Sharifpoor, S.; Cai, K.; Labow, R.S.; Santerre, J.P. Biodegradation and in vivo biocompatibility of a degradable, polar/hydrophobic/ionic polyurethane for tissue engineering applications. Biomaterials 2011, 32, 6034-6044.

10. Blit, P.H.; McClung, W.G.; Brash, J.L.; Woodhouse, K.A.; Santerre, J.P. Platelet inhibition and endothelial cell adhesion on elastin-like polypeptide surface modified materials. Biomaterials 2011, 32, 5790-5800.

11. Lin, S.Y.; Ho, C.J.; Li, M.J. Precision and reproducibility of temperature response of a thermoresponsive membrane embedded by binary liquid crystals for drug delivery. J. Control. Release 2001, 73, 293-301. 
12. Rao, H.; Zhang, Z.; Song, C.; Qiao, T. Polydimethylsiloxane/liquid crystal cross-linked membranes: Preparation, characterization and oxygen transport properties. React. Funct. Polym. 2011, 71, 537-543.

13. Makai, M.; Csanyi, E.; Nemeth, Z.; Palinkas, J.; Eros, I. Structure and drug release of lamellar liquid crystals containing glycerol. Int. J. Pharm. 2003, 256, 95-107.

14. Tu, L.; Li, M.; Mou, S.; Zhou, C. Preparation and blood compatibility of polysiloxane/liquidcrystal composite membranes. Biomaterials 2001, 22, 2595-2599.

15. Lin, S.Y.; Lin, H.L.; Li, M.J. Manufacturing factors affecting the drug delivery function of thermo-responsive membrane prepared by adsorption of binary liquid crystals. Eur. J. Pharm. Sci. 2002, 17, 153-160.

16. Tao, W.; Zhou, H.; Zhang, Y.; Li, G. Novel silsesquioxane mixture-modified high elongation polyurethane with reduced platelet adhesion. Appl. Surf. Sci. 2008, 254, 2831-2836.

17. McEver, R.P.; Beckstead, J.H.; Moore, K.L.; Marshall-Carlson, L.; Bainton, D.F. GMP-140, a platelet alpha granule protein, is also synthesized by vascular endothelium and is located in Weibel-Palade bosies. Clin. Invest. 1989, 84, 92-99.

18. Rand, M.L.; Leung, R.; Packham, M.A. Platelet function assays. Transfus. Apher. Sci. 2003, 28, 307-317.

19. Pan, J.; Li, G.; Chen, Z.; Chen, X.; Zhu, W.; Xu, K. Alternative block polyurethanes based on poly(3-hydroxybutyrate-co-4-hydroxybutyrate) and poly(ethylene glycol). Biomaterials 2009, 30, 2975-2984.

20. Zhou, C.; Yi, Z. Blood-compatibility of polyurethane/liquid crystal composite membranes. Biomaterials 1999, 20, 2093-2099.

21. Dalsin, J.L.; Hu, B.H.; Lee, B.P.; Messersmith, P.B. Mussel adhesive protein mimetic polymers for the preparation of nonfouling surfaces. J. Am. Chem. Soc. 2003, 125, 4253-4258.

22. Knop, K.; Hoogenboom, R.; Fischer, D.; Schubert, U.S. Poly(ethylene glycol) in drug delivery: Pros and Cons as well as potential alternatives. Angew. Chem. Int. Ed. 2010, 49, 6288-6308.

23. Zang, J.; Yuan, J.; Yuan, Y.; Zang, X.; Shen, J.; Lin, S. Platelet adhesive resistance of segmented polyurethane film surface-grafted with vinyl benzyl sulfo monomer of ammonium zwitterions. Biomaterials 2003, 24, 4223-4231.

24. Yuan, J.; Chen, L.; Jiang, X.; Shen, J.; Lin, S. Chemical graft polymerization of sulfobetaine monomer on polyurethane surface for reduction in platelet adhesion. Colloid Surf. B-Biointerfaces 2004, 39, 87-94.

25. Poussard, L.; Burel, F.; Couvercelle, J.P.; Lesouhaitier, O.; Merhi, Y.; Tabrizian, M.; Bunel, C. In vitro thrombogenicity investigation of new water-dispensible polyurethane anionomers bearing carboxylate groups. J. Biomater. Sci.-Polym. Ed. 2005, 16, 335-351.

26. Yuan, J.; Hou, Q.; Liu, B.; Shen, J.; Lin, S. Platelet adhesive resistance of polyurethane surface grafted with zwitterions of sulfobetaine. Colloid Surf. B-Biointerfaces 2004, 36, 19-26.

27. Xu, L.C.; Runt, J.; Siedlecki, C.A. Dynamics of hydrated polyurethane biomaterials: Surface microphase restructuring, protein activity and platelet adhesion. Acta Biomater. 2010, 6, 1938-1947.

28. Dimitrievska, S.; Maire, M.; Diaz-Quijada, G.A.; Robitaille, L.; Ajji, A.; Yahia, L.; Moreno, M.; Merhi, Y.; Bureau, M.N. Low thrombogenicity coating of nonwoven PET fiber structures for vascular grafts. Macromol. Biosci. 2011, 11, 493-502. 
29. Morimoto, N.; Iwasaki, Y.; Nakabayashi, N.; Ishihara, K. Physical properties and blood compatibility of surface-modified segmented polyurethane by semi-interpenetrating polymer networks with a phospholipid polymer. Biomaterials 2002, 23, 4881-4887.

30. Nakabayashi, N.; Williams, D.F. Preparation of non-thrombogenic materials using 2-methacryloyloxyethyl phosphorylcholine. Biomaterials 2003, 24, 2431-2435.

31. Korematsu, A.; Yakemoto, Y.; Nakaya, T.; Inoue, H. Synthesis, characterization and platelet adhesion of segmented polyurethanes grafted phospholipid analogous vinyl monomer on surface. Biomaterials 2002, 23, 263-271.

32. Gorbet, M.B.; Sefton, M.V. Biomaterial-associated thrombosis: Roles of coagulation factors, complement, platelets and leukocytes. Biomaterials 2004, 25, 5681-5703.

33. Spijker, H.T.; Busscher, H.J.; van Oeveren, W. Influence of abciximab on the adhesion of platelets on a shielded plasma gradient prepared on polyethylene. Thromb. Res. 2002, 108, 57-62.

Sample Availability: Not available.

(C) 2011 by the authors; licensee MDPI, Basel, Switzerland. This article is an open access article distributed under the terms and conditions of the Creative Commons Attribution license (http://creativecommons.org/licenses/by/3.0/). 